\title{
Maior carga de gemas da videira resulta em melhora dos índices produtivos e vegetativos da videira 'Cabernet Franc' cultivada em região de elevada altitude
}

\author{
Higher load of grapevine buds results in better yield and vegetative vigor indices in \\ 'Cabernet Franc' grapevine grown in a high-altitude region
}

\author{
Douglas André Würz ${ }^{1 *}$, Bruno Farias Bonin ${ }^{2}$, Alberto Fontanella Brighenti ${ }^{3}$, Adrielen Tamiris \\ Canossa ${ }^{4}$, Juliana Reinehr ${ }^{4}$, Ricardo Allebrandt ${ }^{4}$, Betina Pereira de Bem ${ }^{4}$, Leo Rufato ${ }^{4}$, Aike Anneliese \\ Kretzschmar $^{4}$ \\ ${ }^{1}$ Instituto Federal de Santa Catarina, Canoinhas, SC, Brasil. Autor para correspondência: douglaswurz@hotmail.com \\ ${ }^{2}$ Universidade Federal do Paraná, Curitiba, PR, Brasil. \\ ${ }^{3}$ Universidade Federal de Santa Catarina, Florianópolis, SC, Brasil. \\ ${ }^{4}$ Universidade do Estado de Santa Catarina, Lages, SC, Brasil.
}

Submissão: 18/07/2019 / Aceite: 06/03/2020

\begin{abstract}
RESUMO
Buscando alcançar um adequado balanço vegeto-produtivo, este trabalho teve como objetivo avaliar o efeito do aumento da carga de gemas planta $^{-1}$, no momento da poda, nas características produtivas e equilíbrio vegetativo da videira Cabernet Franc cultivada em região de elevada altitude de Santa Catarina. O presente trabalho foi conduzido durante a safra 2016/2017, em um vinhedo comercial, localizado no munícipio de São Joaquim. Utilizaram-se videiras da cultivar Cabernet Franc enxertadas sobre o portaenxerto 'Paulsen 1103'. Os tratamentos consistiram em quatro diferentes níveis de cargas de gemas: 15, 30,50 e 75 gemas planta $^{-1}$. Avaliou-se no momento da colheita variáveis produtivas e vegetativas, e ao longo do ciclo da videira, avaliou-se o crescimento de ramos e feminelas. O delineamento experimental utilizado foi o de blocos ao acaso, com quatro blocos e cinco plantas por parcela. O aumento da carga de gemas planta $^{-1}$ resultou em aumento do número de ramos e de cachos planta ${ }^{-1}$, resultando em aumento da produção por planta e da produtividade. As variáveis vegetativas foram influenciadas pelo aumento da gemas planta $^{-1}$, observando-se redução do crescimento de ramos e de feminelas. O aumento da carga de gemas possibilitou melhor equilíbrio vegeto-produtivo, com redução do peso de poda, e obtenção do Índice de Ravaz mais adequado para a elaboração de vinhos de qualidade. Em vinhedos que apresentam baixa produtividade e excesso de vigor vegetativo, recomenda-se aumentar a carga de gemas planta $^{-1}$ durante o manejo da poda invernal.
\end{abstract}

PALAVRAS-CHAVE: Vitis vinifera L., produtividade, equilíbrio vegetativo, poda.

\begin{abstract}
To reach an adequate vegetative-productive balance, this work aimed to evaluate the effect of an increase of the bud load.plant ${ }^{-1}$ during pruning, on the productive characteristics and vegetative balance of Cabernet Franc grapevine cultivated in a high-altitude region of the state of Santa Catarina, Brazil. The present work was conducted during the 2016/2017 harvest, in a commercial vineyard located in the municipality of São Joaquim. Cabernet Franc variety grapevines grafted on the 'Paulsen 1103' rootstock were used. The treatments consisted of four different levels of bud loads: 15, 30, 50, and 75 buds.plant $^{-1}$. At the time of harvesting, productive and vegetative variables were evaluated, and the growth of branches and secondary shoots was evaluated along the grapevine cycle. The experimental design was a randomized block design with four blocks and five plants per plot. The increase of the bud load.plant ${ }^{-1}$ increased the $^{2}$ number of branches and bunches.plant ${ }^{-1}$, resulting in increased plant production and productivity. The vegetative variables were influenced by the increase of buds.plant ${ }^{-1}$, with a reduction of the growth of branches and of secondary shoots. The increase of the bud load allowed a better vegetative-productive balance, with the reduction in the weight of pruning, and a Ravaz Index more suitable for the production of quality wines. In vineyards with low productivity and excessive vegetative vigor, the increase of bud load.plant ${ }^{-1}$ during winter pruning management is recommended.
\end{abstract}

KEYWORDS: Vitis vinifera L., productivity, vegetative balance, pruning. 


\section{INTRODUÇÃO}

O estado de Santa Catarina é o segundo maior produtor de uvas (Vitis vinifera L.) do Brasil para vinicultura (MELLO 2015). Ciclos fenológicos mais longos são observados nas regiões de altitude do Estado de Santa Catarina quando comparados com outras regiões produtoras do Brasil. Essas regiões apresentam uma maior disponibilidade de radiação solar e menores temperaturas noturnas na fase final de maturação. Com períodos de maturação mais longos, os vinhedos produzem uvas de maior potencial enológico (MALINOVSKI et al. 2016). Porém, observa-se nessa região elevada disponibilidade hídrica (BEM et al. 2016), solos com altos teores de matéria orgânica (MAFRA et al. 2011), que resultam em crescimento vegetativo excessivo, baixo índice de fertilidade de gemas, podendo afetar a produtividade e a maturação das uvas (BRIGHENTI et al. 2014, WURZ et al. 2017, WÜRZ et al. 2018).

Uma das alternativas para aumentar a produtividade dos vinhedos e melhorar o seu equilíbrio vegetativo seria através da poda de inverno, aumentando a relação gemas planta ${ }^{-1}$. Alterar o número de gemas da videira durante a poda de inverno é a principal e mais barata forma de adequar o rendimento do vinhedo (WINKLER 1965). A poda invernal é uma prática realizada anualmente em regiões de clima temperado, com o principal objetivo de regular a produção temporal da videira (MIELE \& MANDELLI 2012), e ao aumentar a carga de gemas planta $^{-1}$, torna-se possível aumentar a produtividade do vinhedo e melhorar o equilíbrio vegeto-produtivo da videira.

A mudança do número de gemas pode ter efeito a longo prazo, como por exemplo, redução do crescimento dos ramos. Portanto, o aumento do número de gemas pode diminuir o vigor, em relação a aqueles podadas com número menor de gemas (GREVEN et al. 2014). Por exemplo, TROUGHT \& BENNET (2009), sugeriram que o número de gemas pode afetar a taxa de crescimento apical dos ramos.

O número de gemas que permanece nas varas ou nos esporões após a poda determinará a dimensão da área foliar e o número de cachos de uvas. Pesquisas pioneiras realizadas na Nova Zelândia por JACKSON et al. (1984) descreveram que o número de gemas variando de 40 a 150 por plantas, em cinco cultivares, apresentam um comportamento curvilíneo, onde o aumento de 43 para 86 gemas por plantas dobrou a produção, no entanto, ao aumentar o número de gemas para 150 por planta, o aumento da produção foi de apenas $12 \%$ em relação a 86 gemas por planta. Trabalho realizado por GREVEN et al. (2015), com a variedade Sauvignon Blanc, na Nova Zelândia, concluiu que o aumento de 24 para 72 gemas por planta resultou em aumento da produtividade de 4,8 para 12,7 ton ha $^{-1}$.

Os processos adaptativos pelos quais as videiras respondem ao aumento do número de gemas incluem a redução do crescimento vegetativo, redução da fertilidade de gemas, ramos mais curtos com entrenós mais curtos, maior produtividade e maior número de cachos por planta, além de apresentarem cachos mais longos e com bagas menores (CLINGELEFFER 2009).

Buscando alcançar um adequado balanço vegeto-produtivo, este trabalho teve como objetivo avaliar o efeito do aumento da carga de gemas planta $^{-1}$ nas características produtivas e equilíbrio vegetativo da videira Cabernet Franc cultivada em região de elevada altitude de Santa Catarina.

\section{MATERIAL E MÉTODOS}

O presente trabalho foi conduzido durante a safra 2016/2017, em um vinhedo comercial, localizado no

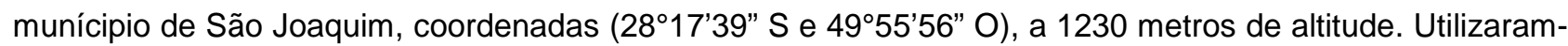
se videiras da cultivar Cabernet Franc enxertadas sobre o porta-enxerto 'Paulsen 1103'. Os vinhedos foram implantados em 2004. O vinhedo caracteriza-se por apresentar plantas espaçadas de 3,0 x 1,5 m, em filas dispostas no sentido N-S, conduzidas em espaldeira, podadas em cordão esporonado duplo, a 1,2 m de altura e cobertas com tela de proteção anti-granizo, e com histórico de baixas produtividades ( 3 ton ha $^{-1}$ ).

O manejo da videira (poda, desbrote, condução, desfolha e colheita) foi realizado com o objetivo de obter maior controle experimental, além de contabilizar o tempo necessário para realizar cada um dos tratos culturais nas diferentes cargas de gemas da videira Cabernet Franc.

Os solos da região se enquadram nas classes Cambissolo Húmico, Neossolo Litólico e Nitossolo Háplico, desenvolvidos a partir de rocha riodacito e basalto (EMBRAPA 2004). O clima da região é classificado como 'Frio, Noites Frias e Úmido', Índice Heliotérmico de 1.714, precipitação pluvial média anual de $1.621 \mathrm{~mm}$ e a umidade relativa do ar média anual de 80\% (TONIETTO \& CARBONNAU 2004).

Os tratamentos consistiram em quatro diferentes níveis de cargas de gemas: 15, 30, 50 e 75 gemas planta $^{-1}$. A poda foi realizada para a Cabernet Franc no dia 08 de Setembro de 2016. Deixou-se 8, 15, 25 esporões com duas gemas cada esporão para os tratamentos 15, 30 e 50 gemas por planta, e para 0 tratamento 75 gemas por planta, deixou-se 30 esporões com duas gemas, e duas varas com 8 gemas cada, sendo portanto, este tratamento podada no sistema de poda mista, caracterizada pela presença de 
esporões e varas.

$\mathrm{Na}$ data da colheita, foram registrados os dados de produção. A produção $(\mathrm{kg})$, o número de cachos e o número de ramos foram registrados para cada planta de cada tratamento. O número de cachos por ramo foi obtido pela divisão do número de cachos por planta pelo número de ramos por planta. Além disso, obteve-se o número de ramos por metro linear de dossel (Ramos $\mathrm{m}^{-1}$ ), através da divisão do número de ramos por planta pelo espaçamento entre plantas. O número de ramos foi obtido se quantificando o número de ramos de quatro plantas por tratamento. Através desses valores foi obtida a \% de brotação, que consiste na relação entre o número de gemas deixadas na poda e do número de gemas que brotaram e originaram ramos.

A produção por planta foi determinada com balança eletrônica de campo, sendo os resultados expressos em kg planta ${ }^{-1}$. A produtividade estimada $\left(\mathrm{t} \mathrm{ha}^{-1}\right)$ foi obtida através da multiplicação da produção por planta pela densidade de plantio (2222 plantas ha ${ }^{-1}$ ).

Para determinar o vigor das plantas, utilizaram-se o peso do material podado e o índice de Ravaz. No momento da poda da videira, em Agosto de 2017, pesou-se os ramos podados de quatro plantas por bloco, com auxílio de uma balança de precisão, resultados expressos em quilograma $(\mathrm{kg})$. O índice de Ravaz foi determinado como uma relação entre o peso dos frutos produzidos e o peso do material podado. A distância de entrenós foi obtida através da relação entre o comprimento do ramo e o número de gemas que cada ramo continha, obtendo-se assim a distância média entrenós do ramo da videira Cabernet Franc.

Avaliou-se também o diâmetro de ramos, com auxílio de um paquímetro digital, e os resultados expressos em milímetros $(\mathrm{mm})$, em dois pontos do ramo: diâmetro do ramo situado na $1^{\mathrm{a}}$ gema e o diâmetro do ramo situado na $10^{\mathrm{a}}$ gema. Além disso, ao longo do ciclo de desenvolvimento vegetativo, avaliou-se o crescimento vegetativo de quatro ramos e quatro feminelas por bloco, com auxílio de uma trena, sendo os resultados expressos em centímetros $(\mathrm{cm})$. A medição do comprimento de ramos e do comprimento de feminelas ocorreu em intervalos de 15 dias. Ao longo do ciclo vegetativo, foram avaliados os mesmos ramos e feminelas.

O delineamento experimental utilizado foi o de blocos ao acaso, com quatro blocos e cinco plantas por parcela. Os dados foram submetidos à análise de variância (ANOVA) e comparados pelo Teste Tukey a $5 \%$ de probabilidade de erro.

\section{RESULTADOS E DISCUSSÃO}

As variáveis vegetativas e produtivas foram influenciadas pelas diferentes cargas de gemas, e estão apresentadas na Tabela 1. Em relação ao número de ramos no momento da colheita, observou-se que os

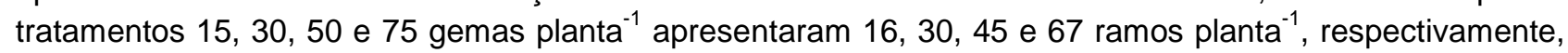
ou seja, houve influência no total de gemas brotadas em relação ao número de gemas deixadas na poda. Com isso, observa-se que ao aumentar o número de gemas por planta na poda, há um decréscimo da \% de brotação. Enquanto as cargas de gemas 15 e 30 gemas planta $^{-1}$ apresentaram uma brotação de $110 \%$ e $101 \%$, respectivamente, e as cargas de gemas de 50 e 75 gemas planta $^{-1}$ apresentam uma brotação de $91 \%$ e $89 \%$, respectivamente (Tabela 1). Apesar de haver uma redução da \% de brotação em função do aumento da carga de gemas planta ${ }^{-1}$, ainda assim, quanto maior a carga de gemas planta ${ }^{-1}$, maior o número de ramos planta $^{-1}$. Da mesma forma, ARCHER \& FOUCHÉ (1987), observaram que a \% de brotação reduziu para $93 \%$ em videiras da variedade Muscat podados com 40 gemas planta $^{-1}$ comparadas a $100 \%$ de brotação em videiras podadas com carga de 16 gemas planta $^{-1}$. Resultados semelhantes também foram observados por GREVEN et al. (2014).

Observou-se que ao aumentar a carga de gemas, houve um aumento da produção e produtividade. Enquanto a poda realizada com 15 gemas planta $^{-1}$ apresentou uma produção de $0,9 \mathrm{~kg} \mathrm{planta}^{-1} \mathrm{e}$ produtividade de 2,1 ton $\mathrm{ha}^{-1}$, as plantas podadas com 75 gemas planta $^{-1}$ apresentaram uma produção de $3,6 \mathrm{~kg} \mathrm{planta}^{-1}$ e produtividade de 8 ton $\mathrm{ha}^{-1}$, ou seja, um aumento de $400 \%$ na produção e de $380 \%$ na produtividade. Ressalta-se que não houve diferenças estatisticamente significativas para a variável índice de fertilidade, que variou de 0,77 a 0,84 cachos ramos $^{-1}$, para as cargas de 15 e 75 gemas planta ${ }^{-1}$, respectivamente.

Os efeitos da carga de gemas planta $^{-1}$ resultam em aumento significativo da produção planta ${ }^{-1}$ e produtividade, corroborando com resultados observados por outros estudos (KURTURAL et al. 2006, BENISMAIL et al. 2007, O'DANIEL et al. 2012). Essa dinâmica de aumento da produtividade pode estar relacionada com o aumento do número de cachos planta $^{-1}$ e massa de cacho, com maior influência do número de cachos planta ${ }^{-1}$, sendo este, de acordo com GREVEN et al. (2014), efeito do maior número de ramos planta ${ }^{-1}$. GREVEN et al. (2015), sugerem que os efeitos da carga de gemas planta $^{-1}$ na produtividade 
estão relacionados ao número de cachos, pois a massa de cachos, a massa e tamanho de bagas são variáveis menos influenciadas pelas alterações do vinhedo.

O aumento da carga de gemas teve efeito na redução do comprimento de ramos e feminelas das plantas da videira Cabernet Franc. Para a variável comprimento de ramo, as cargas de 15 gemas e 30 gemas planta $^{-1}$ apresentaram ramos com 342 e $304 \mathrm{~cm}$ de comprimento, respectivamente. Enquanto que para a carga de 50 gemas planta ${ }^{-1}$, observou-se comprimento de $267 \mathrm{~cm}$. Ressalta-se que o menor comprimento de ramo foi observado nas plantas podadas com 75 gemas planta $^{-1}$, com $217 \mathrm{~cm}$, ou seja, uma redução de $37 \%$. O mesmo comportamento foi observado para a variável comprimento de feminela, onde a carga de 75 gemas planta $^{-1}$ apresentou uma redução de $55 \%$ em relação a carga de 15 gemas planta $^{-1}$, ou seja, uma redução de $65 \mathrm{~cm}$ para $29 \mathrm{~cm}$ de comprimento de feminela. TROUGHT \& BENNETT (2009), sugeriram que a carga de gemas planta ${ }^{-1}$ pode afetar a taxa de crescimento dos ramos principais e secundários, propiciando redução da área foliar total.

Não houve efeito da carga de gemas nas variáveis distância de entrenó, diâmetro de ramo na posição da $1^{\text {a }}$ gema e diâmetro de ramo na posição da $10^{\underline{a}}$ gema (Tabela 1). No entanto, houve influência da carga de gemas nas variáveis peso de poda planta ${ }^{-1}$ e índice de Ravaz. Apesar de apresentar um número maior de ramos, a carga de 75 gemas planta $^{-1}$ apresentou peso de poda de 1,0 kg planta ${ }^{-1}$, enquanto a carga de 15 gemas planta ${ }^{-1}$ apresentou peso de poda de $1,7 \mathrm{~kg}$ planta $^{-1}$, ou seja, mesmo a carga de 75 gemas planta $^{-1}$ apresentar $418 \%$ ramos planta ${ }^{-1}$ a mais que a carga de 15 gemas planta $^{-1}$, o peso de poda foi $70 \%$ inferior nas plantas podadas com 75 gemas planta $^{-1}$. As plantas podadas com 30 e 50 gemas planta ${ }^{-1}$ apresentaram valores intermediários de peso poda, apresentando 1,4 e 1,3 $\mathrm{kg}_{\text {planta }}{ }^{-1}$, respectivamente (Tabela 1).

A carga de 75 gemas planta $^{-1}$ apresentou o maior valor do índice de Ravaz, com 3,4, enquanto a carga de 15 gemas planta $^{-1}$ apresentou o menor valor do índice de Ravaz, com 0,5. Não houve diferenças estatisticamente significativas para as cargas de 30 e 50 gemas planta $^{-1}$, que apresentaram índice de Ravaz de 1,1 e 2,0, respectivamente (Tabela 1). A literatura tem relatado um intervalo no Índice de Ravaz entre 5 a 10 (KLIEWER \& DOKOOZLIAN 2005) para alcançar a maturação da uva.

O dossel vegetativo ideal e o rendimento das videiras podem ser manipulados de várias maneiras, sendo o mais barato aumentar e ajustar a carga de gemas planta ${ }^{-1}$ através da poda, buscando o equilíbrio entre o crescimento vegetativo e a produção. A poda equilibrada, mantendo um número fixo de gemas por unidade de massa de poda, foi sugerida como um método para descrever o número adequado de gemas na poda de inverno (HOWELL 2001), que dentro de sua pesquisa sobre a cultivar Vignoles comprovou que $0,45 \mathrm{~kg}$ de peso de poda de inverno permite que os frutos amadureçam adequadamente na safra seguinte, desenvolvendo um dossel suficientemente capaz de armazenar carboidratos de reserva para a safra seguinte. De acordo com BENISMAIL et al. (2007), a poda equilibrada resultou em melhor equilíbrio no desenvolvimento do dossel vegetativo, reduzindo o crescimento de feminelas.

Tabela 1. Efeito da carga de gemas nas variáveis vegetativas e produtivas da videira Cabernet Franc (Vitis vinifera L.) em região de elevada altitude de Santa Catarina. Safra 2017.

Table 1. Effect of bud load on the vegetative and productive variables of Cabernet Franc (Vitis vinifera L.) grapevine in a high-altitude region of Santa Catarina. 2017 harvest.

\begin{tabular}{|c|c|c|c|c|c|}
\hline & \multicolumn{4}{|c|}{ Carqa de Gemas } & \multirow{2}{*}{$\begin{array}{l}\mathrm{CV} \\
(\%)\end{array}$} \\
\hline & 15 & 30 & 50 & 75 & \\
\hline $\begin{array}{l}2017 \\
\text { Número de Ramos } \\
\text { Brotação (\%) }\end{array}$ & $\begin{array}{c}16 \mathrm{~d} \\
110 \mathrm{a}\end{array}$ & $\begin{array}{c}30 \mathrm{c} \\
101 \mathrm{ab}\end{array}$ & $\begin{array}{l}45 \mathrm{~b} \\
91 \mathrm{~b}\end{array}$ & $\begin{array}{l}67 a \\
89 b\end{array}$ & $\begin{array}{l}3,4 \\
5,8\end{array}$ \\
\hline $\begin{array}{l}\text { Número de Cachos } \\
\left.\text { Produção (kg planta }{ }^{-1}\right)\end{array}$ & $\begin{array}{l}12 \mathrm{~d} \\
0,9 \mathrm{c}\end{array}$ & $\begin{array}{l}23 \mathrm{c} \\
1,5 \mathrm{c}\end{array}$ & $\begin{array}{l}35 \mathrm{~b} \\
2,5 \mathrm{~b}\end{array}$ & $\begin{array}{l}56 \mathrm{a} \\
3,6 \mathrm{a}\end{array}$ & $\begin{array}{c}8,7 \\
18,6\end{array}$ \\
\hline Produtividade (ton ha ${ }^{-1}$ ) & $2,1 \mathrm{c}$ & $3,4 \mathrm{c}$ & $5,6 \mathrm{~b}$ & $8,0 \mathrm{a}$ & 7,1 \\
\hline Comprimento de Ramo $(\mathrm{cm})$ & $342 \mathrm{a}$ & $304 \mathrm{ab}$ & $267 \mathrm{bc}$ & $217 c$ & 11,0 \\
\hline Comprimento de Feminela $(\mathrm{cm})$ & $65 \mathrm{~b}$ & $44 \mathrm{ab}$ & $32 \mathrm{~b}$ & $29 b$ & 32,9 \\
\hline Distância Entrenó (cm) & $6,8 \mathrm{~ns}$ & 7,2 & 6,4 & 6,2 & 13,5 \\
\hline Diâmetro Ramo $1^{\mathrm{a}}$ Gema (cm) & $0,9 \mathrm{~ns}$ & 0,9 & 0,9 & 0,8 & 13,4 \\
\hline Diâmetro Ramo $10^{\mathrm{a}}$ Gema (cm) & $0,8 \mathrm{~ns}$ & 0,8 & 0,7 & 0,6 & 16,2 \\
\hline Peso de Poda (kg) & $1,7 \mathrm{a}$ & $1,4 \mathrm{~b}$ & $1,3 \mathrm{~b}$ & $1,0 \mathrm{c}$ & 6,9 \\
\hline Índice de Ravaz & $0,5 \mathrm{c}$ & $1,1 \mathrm{bc}$ & $2,0 \mathrm{~b}$ & $3,4 \mathrm{a}$ & 25,2 \\
\hline
\end{tabular}

*Médias seguidas da mesma letra, na linha, não diferem entre si pelo teste Tukey a 5\% de probabilidade de erro. ns = não significativo pela análise de variância (ANOVA) a $5 \%$ de probabilidade de erro. 
O crescimento de ramo foi avaliado ao longo do ciclo vegetativo da videira Cabernet Franc para melhor compreensão do efeito da carga de gemas para essa variável (Figura 1). Observa-se que até o dia 10/nov foi semelhante o comprimento de ramo entre os diferentes tratamentos, e após esse período, que compreendeu em média 35 dias após a brotação, o crescimento de ramos das plantas podadas com 15 e 30 gemas planta $^{-1}$ acentuou-se, apresentado picos de crescimento de 08/dez à $12 /$ jan, enquanto, as plantas podadas com 50 e 75 gemas planta $^{-1}$ mantiveram um crescimento de ramo menos acentuada e constante ao longo do ciclo vegetativo.

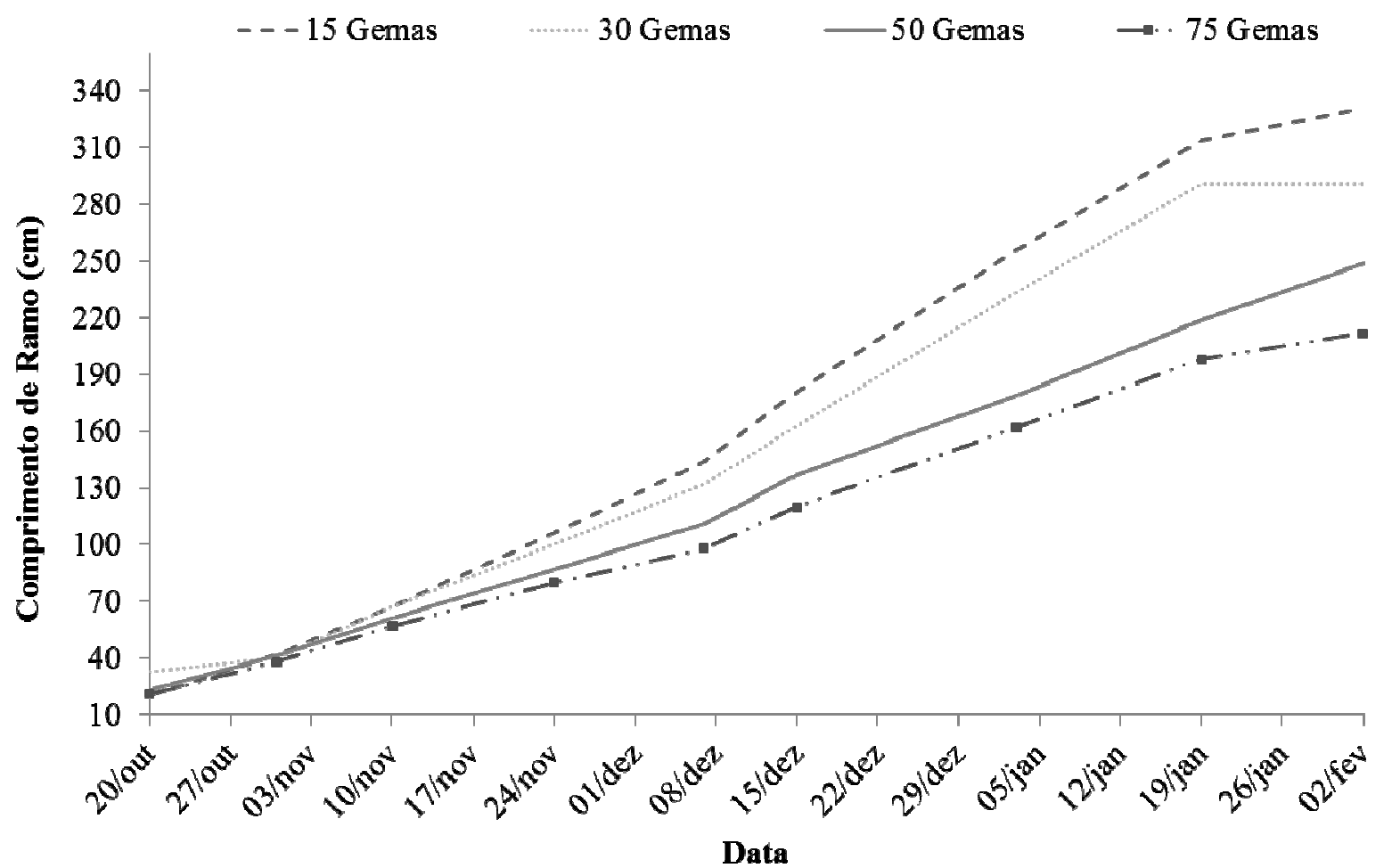

Figura 1. Efeito da carga de gemas no comprimento de ramo da videira Cabernet Franc (Vitis vinifera L.) em região de elevada altitude de Santa Catarina. Safra 2017.

Figure 1. Effect of bud load on the branch length of Cabernet Franc grapevine (Vitis vinifera L.) in a highaltitude region of Santa Catarina. 2017 harvest.

O comportamento ao longo do ciclo vegetativo do comprimento de feminela está descrito na Figura 2. As avaliações iniciam no dia 07/dez e encerraram no dia 15/fev. Observou-se até o dia 04/jan comportamento semelhante do aumento do comprimento de feminela, e a partir desta data, houve um aumento do comprimento de feminela para as cargas de 15 e 30 gemas planta $^{-1}$. Para as cargas de 50 e 75 gemas $_{\text {planta }}{ }^{-1}$, observou-se um aumento mais acentuado a partir do dia 18/jan, contudo, apresentando valores inferiores aos observados pelas cargas de 50 e 75 gemas planta $^{-1}$ (Figura 2).

Para BENISMAIL et al. (2007), a baixa carga de gemas planta ${ }^{-1}$, resulta em efeitos no crescimento vegetativo, com dominância do crescimento dos ramos principais e de feminelas, provocando um desequilíbrio vegetativo a plantas submetidas a poda com baixa carga de gemas.

A busca pela melhor relação produção e qualidade apresenta dificuldades, ainda mais nas condições de altitude onde o clima úmido e solo fértil tornam o controle do crescimento um desafio. No entanto, plantas desequilibradas, produzem mostos desbalanceados, resultando em um vinho de pouca qualidade (JACKSON \& LOMBARD 1993).

Os resultados das variáveis vegeto-produtivas mostram claramente o efeito da manipulação da carga de gemas planta ${ }^{-1}$ nas características do dossel vegetativo, resultando em alteração do comprimento de ramo, comprimento de feminela, e na relação área foliar produção ${ }^{-1}$, menor peso de poda e maiores produtividades. 


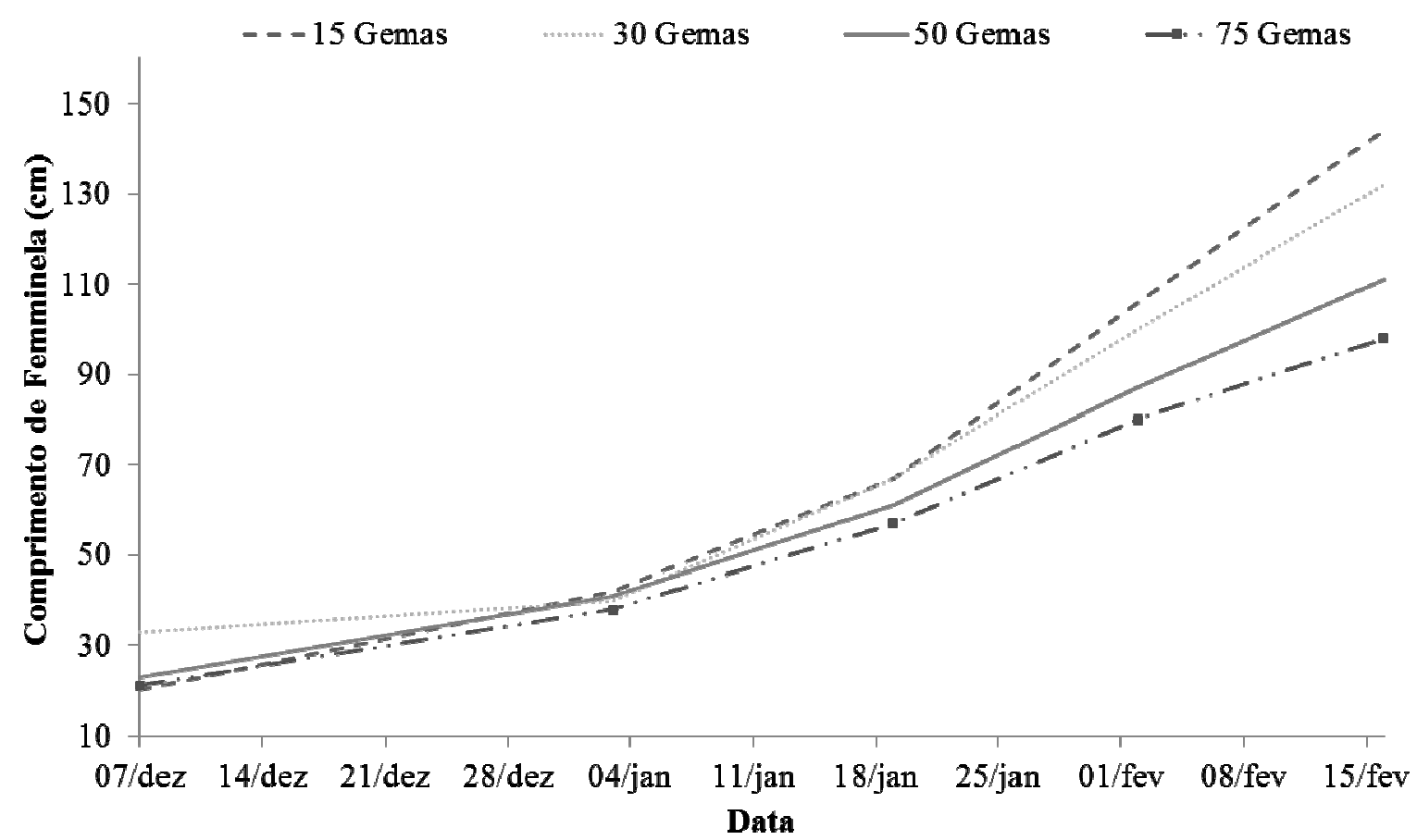

Figura 2. Efeito da carga de gemas no comprimento de feminela da videira Cabernet Franc (Vitis vinifera L.) em região de elevada altitude de Santa Catarina. Safra 2017.

Figure 2. Effect of the bud load on the length of females of the Cabernet Franc (Vitis vinifera L.) grapevine in high altitude region of Santa Catarina. 2017 season.

\section{CONCLUSÃO}

O aumento da carga de gemas planta ${ }^{-1}$ resultou em aumento do número de ramos e de cachos planta $^{-1}$, resultando em aumento da produção planta e da produtividade.

As variáveis vegetativas foram influenciadas pelo aumento do número de gemas planta ${ }^{-1}$, observando-se redução do crescimento de ramos e de feminelas.

O aumento da carga de gemas possibilitou melhor equilíbrio vegeto-produtivo, com redução do peso de poda, e obtenção do Índice de Ravaz mais adequado para a elaboração de vinhos de qualidade.

Em vinhedos que apresentam baixa produtividade e excesso de vigor vegetativo, recomenda-se aumentar a carga de gemas planta ${ }^{-1}$ durante o manejo da poda de inverno.

\section{REFERÊNCIAS}

ARCHER E \& FOUCHÉ GW. 1987. Effect of bud load and rootstock cultivar on the performance of $V$. vinifera L. cv. Red Muscadel (Muscat noir). South African Journal for Enology and Viticulture 8: 6-10.

BEM BP et al. 2016. Effect of four training systems on the temporal dynamics of downy mildew in two grapevine cultivarws in southern Brazil. Tropical Plant Pathology 41: 370-379.

BENISMAIL MC et al. 2007. Effect of Bud Load and Canopy Managemant on Growth and Yield Components of Grape cv. 'Cardinal' under mild climatic conditions os Agadir Are of Morocco. Acta Horticulturae 754: 197-204.

BRIGHENTI AF et al. 2014. Desempenho vitícola de variedades autóctones italianas em condição de elevada altitude no Sul do Brasil. Pesquisa Agropecuária Brasileira 49: 465-474.

CLINGELEFFER PR. 2009. Influence of canopy management systems on vine productivity and fruit composition. In:

Recent Advances in Grapevine Canopy Management. Davis: University of California. p.13-19.

EMBRAPA. 2004. Empresa Brasileira de Pesquisa Agropecuária. Solos do Estado de Santa Catarina. Rio de Janeiro: Embrapa Solos. 726p. (Embrapa Solos. Boletim de Pesquisa e Desenvolvimento 46).

GREVEN MM et al. 2014. Influence of retained node number on Sauvignon Blanc grapevine vegetative growth and yield. Australian Journal of Grape and Wine Research 20: 263-271.

GREVEN MM et al. 2015. Influence of retained node number on Sauvignon Blanc grapevine phenology in a cool climate. Australian Journal of Grape and Wine Research 21: 209-301.

HOWELL GS. 2001. Sustainable grape productivity and the growth-yield relationship: A review. American Journal of Enology and Viticulture 52: 165-174.

JACKSON DI et al. 1984. Vine response to increased node numbers. American Journal of Enology and Viticulture 35: 161-163.

JACKSON DI \& LOMBARD PB 1993. Environmental and management practices affecting grape composition and wine quality - a review. American Journal of Enology and Viticulture 44: 409-430. 
KLIEWER WM \& DOKOOZLIAN NK 2005. Leaf area/crop weight ratios of grapevines: Influence on fruit composition and wine quality. American Journal of Enology and Viticulture 56: 170-181.

KURTURAL SK et al. 2006. Effects of pruning and cluster thinning on yield and fruit composition of 'Chambourcin' grapevines. HortTechnology 16: 233-240.

MAFRA SHM et al. 2011. Atributos químicos do solo e estado nutricional de videira Cabernet Sauvignon (Vitis vinifera L.) na Serra Catarinense. Revista de Ciências Agroveterinárias 10: 44-53.

MALINOVSKI LI et al. 2016. Viticultural performance of Italian grapevines in high altitude regions of Santa Catarina State, Brazil. Acta Horticulturae 1115: 203-210.

MELLO LMR. 2015. Vitivinicultura Brasileira: Panorama 2014. Bento Gonçalves: Embrapa Uva e Vinho. 6p. (Comunicado Técnico 175).

MIELE A \& MANDELLI F. 2012. Manejo do dossel vegetativo e seu efeito nos componentes de produção da videira Merlot. Revista Brasileira de Fruticultura 34: 964-973.

O'DANIEL SB et al. 2012. Effects of balanced pruning severity on Traminette (Vitis spp.) in a warm climate. American Journal of Enology and Viticulture 63: 284-290.

TONIETTO J \& CARBONNEAU A 2004. A multicriteria climatic classification system for grape-growing regions worlwide. Agricultural and Forest Meteorology 124: 81-97.

TROUGHT M \& BENNETT J 2009. Influence of training system on Sauvignon blanc grapevine performance 2004-2009. Report to New Zealand Winegrowers. Auckland: New Zealand Winegrowers.

WINKLER AJ. 1965. Viticultura. México: Continental. 792p.

WÜRZ DA et al. 2017. Agronomic performance of 'Cabernet Sauvignon' with leaf removal management in a high-altitude region of Southern Brazil. Pesquisa Agropecuária Brasileira 52: 869-876.

WÜRZ DA et al. 2018. Época de desfolha e sua influência no desempenho vitícola da uva 'Sauvignon Blanc' em região de elevada altitude. Revista de Ciências Agroveterinárias 17: 91-99. 